\title{
On performance of analog network coding in the presence of phase noise
}

\author{
Amir Ligata1*, Haris Gacanin ${ }^{2}$ and Fumiyuki Adachi ${ }^{3}$
}

\begin{abstract}
Analog network coding (ANC) as a simple implementation of physical layer network coding based on orthogonal frequency division multiplexing (OFDM) has been proposed to increase the network capacity and reliability of bi-directional link between a pair of users. In ANC protocol, an information between a pair of users is exchanged through two orthogonal time phases (i.e., multiple-access and broadcast phases). On the other hand, the phase noise (PN) introduces phase offset and inter carrier interference (ICI) to the useful signal. Thus, in ANC scheme PN will affect the useful signal during both multiple-access and broadcast phases. In this article, we present a performance analysis of ANC scheme using OFDM in the presence of PN in frequency-selective fading channel. We derive the total composite variance of ANC scheme in the presence of PN to obtain the signal-to-interference-plus-noise ratio (SINR) expression. Then, we evaluate the system's performance in terms of bit error rate (BER), SINR degradation, and ergodic capacity through both numerical and computer simulations. Computer simulated average BER has been consistent with the numerical results, validating the presented analysis. Our results have shown that the ANC scheme is more sensitive to the PN introduced during the broadcast phase (i.e., at destination) than during the multiple-access phase (i.e., at relay). This is because of the higher $\mathrm{ICl}$ to the useful signal and enhanced noise due to the imperfect self-information removal at the destination. In addition, the performance degradation of ANC scheme based on OFDM in the presence of PN is highly expressed for the PN linewidth values up to $20 \mathrm{~Hz}$.
\end{abstract}

\section{Introduction}

Although the wireless traffic demands driven by the users requirements continuously grow, available radio resources remain fixed. Intensive effort is being made in order to exploit the limited radio spectrum more efficiently and minimize the mutual interference caused by the multiuser access. Orthogonal frequency division multiplexing (OFDM) is considered to be an attractive transmission technique for broadband communications over a wireless channel. A simple implementation of physical layer network coding, known as analog network coding (ANC) using OFDM, has been proposed to increase the network capacity and reliability of bi-directional communication between a pair of users using the same channel [1-4]. In $\mathrm{ANC}$, an information between a pair of users is exchanged through two orthogonal time phases; (i) in multiple-access phase both users simultaneously transmit towards the

\footnotetext{
*Correspondence: amir.ligata@ipsa-institut.com

${ }^{1}$ Communication Department, IPSA Institute, Sarajevo, Bosnia and Herzegovina

Full list of author information is available at the end of the article
}

relay, and (ii) in broadcast phase the relay broadcast the received signal to both users using amplify-and-forward $(\mathrm{AF})$ protocol. The phase noise $(\mathrm{PN})$ caused by imperfections of the local oscillators rotates all subcarriers in OFDM by a common phase error (CPE) and introduces inter-carrier interference (ICI) to the useful signal which degrades the bit error rate (BER) performance [5-13]. The problem of phase synchronization in relaying based on $\mathrm{AF}$ protocol is investigated in [14], and it was shown that the relay's PN has only limited impact on the system's performance. Implementation of ANC scheme in frequencydomain was presented in [15], and carrier frequency offset compensation is considered at relay to capture the effect of PN in ANC scheme. In [16], impact of ICI on cooperative SFBC-OFDM is theoretically analyzed and an iterative algorithm for inter-symbol interference (ISI) and ICI cancelation is proposed. It was showed that the two-step cancelation algorithm can efficiently suppress the ICI and ISI. Cooperative STBC-OFDM system with imperfect frequency synchronization is investigated in [17], and an

\section{Springer}

(c) 2013 Ligata et al: licensee Springer. This is an Open Access article distributed under the terms of the Creative Commons Attribution License (http://creativecommons.org/licenses/by/2.0), which permits unrestricted use, distribution, and reproduction in any medium, provided the original work is properly cited. 
ICI mitigation algorithm was proposed which considerably improves the system's symbol error rate. In the case of imperfect channel state information (CSI), the performance of ANC scheme in the presence of PN would be further degraded as the noise due to the imperfect-self information removal and CE error would arise [18-20]. We note here that the sampling frequency offset which occurs due to non-synchronized sampling at the transmitter and receiver clock may also degrade the performance of ANC scheme as the ICI arises and the OFDM symbol is rotated [21].

In ANC communication protocol, the transmission is done over two orthogonal phases in a time division multiplexing (TDM) fashion. This way, the PN is introduced at the reception in each transmission phase (i.e., multipleaccess and broadcast phases), and consequently may cause $\mathrm{CPE}$ and ICI to the useful signal at the receiver end (i.e., relay and destination). Thus, the BER performance of ANC based on OFDM may be degraded. To the best of the authors' knowledge, an impact of PN on the performance of ANC scheme has not been addressed in the literature.

In this article, we theoretically analyze the impact of $\mathrm{PN}$ on the performance of OFDM-based ANC scheme in frequency-selective fading channel. We derive the total composite noise variance of ANC scheme in the presence of $\mathrm{PN}$, while approximating $\mathrm{PN}$ as a zero-mean Wiener process to obtain the signal-to-interference-plusnoise ratio (SINR) expression. The BER performance is evaluated by the way of both numerical and computer simulations. The performance of ANC scheme in the presence of $\mathrm{PN}$ is further evaluated through numerical simulation of SINR degradation and ergodic capacity. Our simulation results indicate that the performance of ANC scheme is more sensitive to the PN introduced at the reception during the broadcast phase (i.e., destination), since the corresponding PN affects the self-information removal process at the destination.

This article is organized as follows. Section 2 gives an overview of the network model. The performance analysis of ANC in the presence of PN is presented in Section 3. Numerical results and discussion are given in Section 4. Section 5 concludes the article.

\section{Network model}

In this section, first we introduce a PN model and then the ANC network model in the presence of $\mathrm{PN}$ is presented. In our analysis, $T_{c}$ discrete-time representation is used, with $T_{c}$ being the fast Fourier transform (FFT) sampling period.

\subsection{PN model}

PN has been modeled as Wiener process in most of the previous works [5-13], and it is proved to be sufficiently accurate [6]. Modeling PN as Wiener process is suitable for evaluating the impact of $\mathrm{PN}$ on a system performance by analytical methods. Note that PN can also be modeled by nonlinear process as presented in [22] but we adopt the former model due to the mathematical trackability. PN given by $\phi(t)$ is defined as a difference between the phase of the receiver oscillator and the phase of the carrier of the received signal, and occurs due to imperfections of the local oscillators. PN at receiver's (i.e., relay or destination) local oscillator introduces a random phase rotation of $e^{j \phi(t)}$ to the received signal in the time domain. $\mathrm{PN}$ is given by

$$
\phi(t)=\phi(t-1)+\delta,
$$

for $t=1, \ldots, N_{c}-1$, with $N_{c}$ being the number of subcarriers. In (1), $\delta$ denotes independent Gaussian complex variable with zero-mean and the variance $2 \sigma_{\phi}^{2}=4 \pi \beta|t|$, with $\beta[\mathrm{Hz}]$ being the single-sided 3-dB linewidth of the Lorentzian power density spectrum [5]. Note that the initial phase $\phi(0)$ is uniformly distributed over $[0,2 \pi][8]$. $\mathrm{PN}$ represented in frequency-domain is given by

$$
\alpha(n)=\frac{1}{N_{c}} \sum_{t=0}^{N_{c}-1} e^{j \phi(t)} \exp \left(-j 2 \pi n \frac{t}{N_{c}}\right)
$$

for $n=0, \ldots, N_{c}-1$. PN affects the received signal by attenuating and rotating the useful signal, and its impact on the OFDM system is reflected through CPE and ICI [8]. Thus, in the presence of PN in OFDM-based system all subcarriers of the signal are rotated by a CPE, while ICI causes a blurring of the constellation like thermal noise.

\subsection{ANC network model}

Network model as illustrated in Figure 1 consists of a relay $R$ and two mobile terminals $U_{0}$ and $U_{1}$. We assume that the mobile terminals are out of each others transmission range while both of them are in the relay coverage area, and thus direct link between the mobile terminals does not exist. The scheme with the direct link with uni-directional transmission between two mobile terminals is discussed in [23,24]. Bi-directional communication between two users $U_{0}$ and $U_{1}$ is done through two orthogonal time phases.

During the first time phase, both users simultaneously transmit its information towards the relay (multipleaccess phase), while in the second time phase relay broadcasts the received signal using AF protocol (broadcast phase).

\subsubsection{Multiple-access phase}

During the multiple-access phase, both users simultaneously transmit its information towards relay, where the $j$ th $(j=0,1)$ user's information is given by $\left\{d_{j}(n) ; n=\right.$ $\left.0, \ldots, N_{c}-1\right\}$ with $E\left[\left|d_{j}(n)\right|^{2}\right]=1$, and $E[\cdot]$ being ensemble average operation. The time-domain signal $\left\{s_{j}(t) ; t=\right.$ 


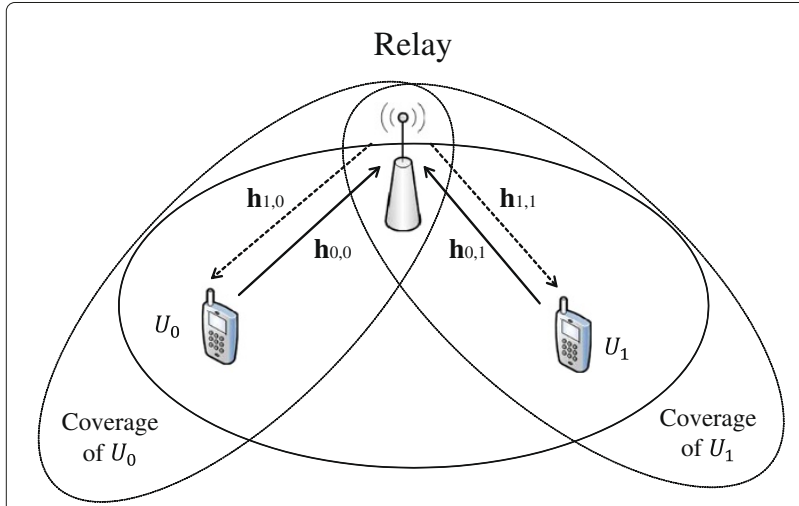

Figure 1 ANC network model.

$\left.0, \ldots, N_{c}-1\right\}$ denotes the IFFT of the $j$ th user's datamodulated symbol sequence $\left\{d_{j}(n)\right\}$. Then, a guard interval (GI) length of $N_{g}\left(N_{g}>L\right)$ is inserted, where $L$ denotes the number of channel paths. Finally, the signal is transmitted over a frequency-selective fading channel.

Wireless channel is characterized with discrete-time channel impulse response $h_{m, j}(t)=\sum_{l=0}^{L-1} h_{l, m, j} \delta\left(t-\tau_{l}\right)$, where $L, h_{l, m, j}, \tau_{l}$ and $\delta(t)$, respectively, denote the number of paths, the path gain between the $j$ th terminal and the relay during $m$ th $(m=0,1)$ stage, the $l$ th path normalized by IFFT sampling period, and the Dirac delta function. $\left\{h_{l, m, j} ; l=0, \ldots, L-1\right\}$ are zero-mean independent complex variables with variance $E\left[\left|h_{l, m, j}\right|^{2}\right]=1 / L$. Without loss of generality, we assume $\tau_{0}=0<\tau_{1}<\cdots<\tau_{L-1}$, where the $l$ th path delay is given by $\tau_{l}=l \Delta$, with $\triangle(\geq 1)$ being the time delay separation between adjacent paths.

The received signal represented in the time-domain at the relay during the multiple-access phase is given by

$$
r_{r}(t)=\sqrt{P_{s}} e^{j \phi_{r}(t)} \sum_{j=0}^{1}\left[s_{j}(t) \otimes h_{0, j}(t)\right]+n_{r}(t)
$$

for $t=0, \ldots, N_{c}-1$, where $\otimes, n_{r}(t)$, and $h_{0, j}(t)$, respectively, denote the circular convolution operation, the additive white Gaussian noise (AWGN) at the relay with power spectral density $N_{0}$, and the channel impulse response between the $j$ th user and the relay during multiple-access phase. In (3), $P_{s}\left(=E_{s} / T_{c}\right)$ denotes the power coefficient with $E_{s}$ being the data-modulated symbol energy, while $\phi_{r}(t)$ represents the PN introduced at the relay. The received signal in frequency-domain is given by

$$
\begin{aligned}
R_{r}(n)= & \sqrt{P_{s}} \alpha_{r}(0) \sum_{j=0}^{1}\left[d_{j}(n) H_{0, j}(n)\right] \\
& +\Theta_{r}(n)+N_{r}(n),
\end{aligned}
$$

for $n=0, \ldots, N_{c}-1$, where the first, the second, and the third terms, respectively, denote the desired signal, the
ICI due to PN at the relay, and the AWGN. In (4), $\alpha_{r}(0)$ and $\Theta_{r}(n)$, respectively, denote the CPE component of the $\mathrm{PN}$ at the relay defined with (2), and the ICI at the $n$th frequency component introduced at the relay, and is given by

$$
\Theta_{r}(n)=\sqrt{P_{s}} \sum_{k=0, k \neq n}^{N_{c}-1} \alpha_{r}(n-k) \sum_{j=0}^{1} d_{j}(k) H_{0, j}(k),
$$

where $\alpha_{r}(n)$ denotes the $n$th component of the PN at the relay defined with (2).

\subsubsection{Broadcast phase}

The received signal at relay is normalized by a factor $\sqrt{1 / E\left[\left|R_{r}(n)\right|^{2}\right]}$, and before forwarding, the normalized signal is amplified with factor $\sqrt{P_{s}}$. During the reception at $j$ th user, due to imperfections of local oscillator, PN causes rotation of the received signal by $e^{j \phi_{j}(t)}$. Thus, the signal received at $j$ th destination represented in the time-domain is given by

$$
r_{j}(t)=e^{j \phi_{j}(t)}\left[r_{r}(t) \otimes h_{1, j}(t)\right]+n_{j}(t)
$$

for $t=0, \ldots, N_{c}-1$, where $n_{j}(t)$ denotes AWGN at the $j$ th user. After removing the GI, an $N_{c}$-point FFT is applied to decompose the received signal into $N_{c}$ frequency components given by

$$
\begin{aligned}
R_{j}(n)= & \sqrt{P_{s}} \alpha_{j}(0) \alpha_{r}(0)\left[\sum_{j=0}^{1} d_{j}(n) H_{0, j}(n)\right] H_{1, j}(n) \\
& +\alpha_{j}(0) \Theta_{r}(n) H_{1, j}(n) \\
& +\alpha_{j}(0) N_{r}(n) H_{1, j}(n) \\
& +\Theta_{j}(n)+N_{j}(n),
\end{aligned}
$$

where $\Theta_{j}(n)$ denotes the ICI due to PN caused by local oscillator of the $j$ th user at $n$th component and is given by

$$
\Theta_{j}(n)=\sum_{k=0, k \neq n}^{N_{c}-1} \alpha_{j}(n-k) R_{r}(k) H_{1, j}(k)
$$

for $n=0, \ldots, N_{c}-1$.

After the $j$ th user $U_{j}$ self-information removal (i.e., $\left.\sqrt{P_{s}} d_{j}(n) H_{0, j}(n) H_{1, j}(n)\right)$ the received signal can be represented as

$$
\begin{aligned}
\tilde{R}_{j}(n)= & \sqrt{P_{s}} \alpha_{j}(0) \alpha_{r}(0) d_{\bar{j}}(n) H_{0, j}(n) H_{1, j}(n) \\
& +\sqrt{P_{s}}\left[\alpha_{j}(0) \alpha_{r}(0)-1\right] d_{j}(n) H_{0, j}(n) H_{1, j}(n) \\
& +\alpha_{j}(0) \Theta_{r}(n) H_{1, j}(n) \\
& +\alpha_{j}(0) N_{r}(n) H_{1, j}(n) \\
& +\Theta_{j}(n)+N_{j}(n)
\end{aligned}
$$


where the bar over the expression signifies the unitary complement operation (i.e., 'NOT' operation) that performs logical negation of the value under the bar, with $\bar{j} \in\{0,1\}$.

Finally, one tap frequency-domain equalization (FDE) is applied to combat the effects of frequency-selective channel as $\hat{d}_{j}(n)=\tilde{R}_{j}(n) w_{j}(n)$ for $n=0, \ldots, N_{c}-1$, where the equalization weight is given by $w_{j}(n)=$ $H_{0, \bar{j}}^{*}(n) H_{1, j}^{*}(n) /\left|H_{0, j}(n) H_{1, j}(n)\right|^{2}[4]$.

\section{Performance analysis}

In this section, we present derivation of the composite noise variance, and then expression for the SINR degradation is given. We note in this article that no path loss and shadowing are assumed. However, the general conclusion remains irrespective of the assumption regarding the path loss and shadowing. Moreover, we assume perfect knowledge of the CSI. The performance of ANC scheme with imperfect CSI in the presence of PN would be degraded. The performance of ANC with imperfect CSI has been considered in [18-20], while the joint analysis of $\mathrm{PN}$ and imperfect CSI would be difficult if not impossible to derive.

\subsection{Composite noise variance}

Using (7) the decision variable at $j$ th user after the equalization is given by

$$
\begin{aligned}
\hat{d}_{j}(n)= & \sqrt{P_{s}} \alpha_{j}(0) \alpha_{r}(0) d_{\bar{j}}(n) \hat{H}_{j}(n)+\xi_{j}(n)+\mathcal{C}_{r}(n) \\
& +\mathcal{C}_{j}(n)+\mathcal{N}(n),
\end{aligned}
$$

where $\xi_{j}(n), \mathcal{C}_{r}(n), \mathcal{C}_{j}(n)$, and $\mathcal{N}(n)$, respectively, denote the $n$th subcarrier of the random self-interference, the ICI at the relay due to $\mathrm{PN}$ at relay, the ICI at the destination due to PN at the destination, and the AWGN, and are given by

$$
\begin{aligned}
\xi_{j}(n) & =\sqrt{P_{s}}\left[\alpha_{j}(0) \alpha_{r}(0)-1\right] d_{j}(n) \hat{H}_{j}(n) \\
\mathcal{C}_{r}(n) & =\alpha_{j}(0) \Theta_{r}(n) \hat{H}_{1, j}(n) \\
\mathcal{C}_{j}(n) & =\Theta_{j}(n) \\
\mathcal{N}(n) & =\alpha_{j}(0) N_{r}(n) H_{1, j}(n)+\hat{N}_{j}(n)
\end{aligned}
$$

for $n=0, \ldots, N_{c}-1$, with $\hat{H}_{j}(n)=H_{0, j}(n) H_{1, j}(n) w_{j}(n)$ and $\hat{H}_{1, j}(n)=H_{1, j}(n) w_{j}(n)$. It can be seen from (11) that due to PN introduced at the relay and $j$ th user, perfect selfinformation removal is not possible.

We assume that the modulated data-symbols, the PN, the channel coefficients, and AWGN are independent of each other. Consequently, the terms given by (11) are independent random variables. Thus, the variance of the composite noise (i.e., the sum of the noise due to imperfect self-information removal, the ICI at relay, the ICI at destination, and the AWGN) is represented by $2 \sigma^{2}=$ $E\left[\left|\xi_{j}(n)\right|^{2}\right]+E\left[\left|\mathcal{C}_{r}(n)\right|^{2}\right]+E\left[\left|\mathcal{C}_{j}(n)\right|^{2}\right]+E\left[|\mathcal{N}(n)|^{2}\right]$.

By using (5), (8) in (11), variance of the composite noise $2 \sigma^{2}$ can be represented as

$$
\begin{aligned}
2 \sigma^{2}= & P_{s} E\left[\left|\alpha_{j}(0) \alpha_{r}(0)-1\right|^{2}\right]\left|\hat{H}_{j}(n)\right|^{2} \\
& +P_{s} E\left[\left|\alpha_{j}(0) \Theta_{r}(n)\right|^{2}\right]\left|\hat{H}_{1, j}(n)\right|^{2} \\
& +E\left[\left|\Theta_{j}(n)\right|^{2}\right] \\
& +E\left[\left|\alpha_{j}(0) N_{r}(n)\right|^{2}\right]\left|H_{1, j}(n)\right|^{2}+E\left[\left|\hat{N}_{j}(n)\right|^{2}\right] .
\end{aligned}
$$

In order to calculate the variance of the composite noise given by (12), we have to evaluate the variance of PN term, the ICI at the relay and ICI at the destination.

\subsubsection{PN term}

Variance of the PN term is given by [9]

$$
\begin{aligned}
\Upsilon_{r(j)}(k) & =E\left[\left|\alpha_{r(j)}(k)\right|^{2}\right] \\
& =\frac{1}{N_{c}^{2}}\left[\frac{\rho(k)^{N_{c}+1}-\left(N_{c}+1\right) \rho(k)+N_{c}}{(\rho(k)-1)^{2}}-N_{c}\right],
\end{aligned}
$$

where $\rho(k)=e^{j\left(2 \pi k / N_{c}\right)-\left(\pi \beta_{r(j)} T_{s} / N_{c}\right)}$. We note that by substituting $k=0$ in (13) variance of the CPE is obtained. For small PN encountered in practice, (13) can be simplified by approximating the exponential term with Taylor series. This assumption stands if the variance of the $\mathrm{PN}$ is much less then unity (i.e., $\sigma_{\phi}^{2} \ll 1$ ) which is the case in practice. Then, variance of the PN term can be represented as

$$
\Upsilon_{r(j)}(k)= \begin{cases}1-\frac{\pi \beta_{r(j)} T_{s}}{3} & \text { for } k=0 \\ \frac{\pi \beta_{r(j)}}{3} & \text { otherwise, }\end{cases}
$$

where $T_{s}\left(=N_{c} T_{c}\right)$ denotes the symbol length duration.

\subsubsection{ICl at the relay}

Now, we calculate the variance of the ICI introduced at the relay. By using (5) and (13) we obtain

$$
E\left[\left|\Theta_{r}(n)\right|^{2}\right]=P_{s} \sum_{k=0, k \neq n}^{N_{c}-1} \Upsilon_{r}(k)\left[\sum_{j=0}^{1}\left|H_{0, j}(k)\right|^{2}\right] .
$$

\subsection{3 $\mathrm{ICl}$ at the destination}

By using (4) and (8) we calculate the variance of the ICI at the destination as

$$
\begin{aligned}
E\left[\left|\Theta_{j}(n)\right|^{2}\right]= & \sum_{k=0, k \neq n}^{N_{c}-1} \Upsilon_{j}(k)\left[P_{s} \Upsilon_{r}(0) \sum_{j=0}^{1}\left|H_{0, j}(n)\right|^{2}\right. \\
& \left.+E\left[\left|\Theta_{r}(n)\right|^{2}\right]+E\left[\left|N_{r}(n)\right|^{2}\right]\right]\left|H_{1, j}(k)\right|^{2} .
\end{aligned}
$$


By substituting (14), (15), and (16) in (12) we obtain the variance of the composite noise given by

$$
\begin{aligned}
2 \sigma^{2}= & \frac{E_{s}}{2 N_{0}}\left[\frac{\pi \beta_{r} T_{s}}{3}+\frac{\pi \beta_{j} T_{s}}{3}-\left(\frac{\pi T_{s}}{3}\right)^{2} \beta_{r} \beta_{j}\right]\left|\hat{H}_{j}(n)\right|^{2} \\
& +\frac{E_{s}}{2 N_{0}}\left(1-\frac{\pi \beta_{j} T_{s}}{3}\right)\left(\frac{\pi \beta_{r} T_{s}}{3}\right)\left\{\sum_{k=0, k \neq n}^{N_{c}-1}\right. \\
& \left.\times\left[\sum_{j=0}^{1}\left|H_{0, j}(k)\right|^{2}\right]\left|\hat{H}_{1, j}(n)\right|^{2}\right\}+\frac{E_{s}}{2 N_{0}}\left(\frac{\pi \beta_{j} T_{s}}{3}\right) \\
& \times\left(\frac{\pi \beta_{r} T_{s}}{3}\right)\left\{\sum_{k=0, k \neq n}^{N_{c}-1}\left[\sum_{m=0, m \neq k}^{N_{c}-1} \sum_{j=0}^{1}\left|H_{0, j}(m)\right|^{2}\right]\right. \\
& \times\left(1-\frac{\pi \beta_{r} T_{s}}{3}\right)\left\{\sum_{k=0, k \neq n}^{N_{c}-1}\left[\sum_{j=0}^{1}\left|H_{0, j}(k)\right|^{2}\right]\left|H_{1, j}(k)\right|^{2}\right\} \\
& \times\left(\frac{\pi \beta_{j} T_{s}}{3}\right)\left[\sum_{k=0, k \neq n}^{N_{c}-1}\left|H_{1, j}(k)\right|^{2}\right]+\left(1-\frac{E_{s}}{2 N_{0}}\left(\frac{\pi \beta_{j} T_{s}}{3}\right)\right. \\
& +1 .
\end{aligned}
$$

We observe from (17) that the impact of the PN on the composite noise variance can be seen through noise due to imperfect self-information removal, the ICI to the useful signal at the relay and at the destination and the AWGN, in that order.

Finally, the SINR expression for the OFDM-based ANC scheme in the presence of $\mathrm{PN}$ at the $j$ th user $U_{j}$ is given by

$\gamma_{j}^{P N}\left[\frac{E_{s}}{N_{0}}, \beta_{o}\right]=\frac{\left(\frac{E_{s}}{2 N_{0}}\right)\left(1-\frac{\pi \beta_{r} T_{s}}{3}\right)\left(1-\frac{\pi \beta_{j} T_{s}}{3}\right)\left|\hat{H}_{j}(n)\right|^{2}}{2 \sigma^{2}}$, where $\beta_{o}=\beta_{r}, \beta_{j}$.

\subsection{SINR degradation}

In order to evaluate the impact of ICI on useful signal caused by $\mathrm{PN}$ at relay and destination we investigate the SINR degradation. The SINR degradation $D$ is defined as

$$
D=-10 \log _{10}\left(\frac{\gamma_{j}}{\gamma_{j}^{P N}}\right)
$$

where $\gamma_{j}$ denotes the SINR expression of ANC in the absence of PN [4]. By using (18), the SINR degradation for ANC scheme in the presence of $\mathrm{PN}$ is given by

$$
\begin{aligned}
D= & -10 \log _{10} \\
& \times\left(\frac{E\left[\left|\xi_{j}(n)\right|^{2}\right]+E\left[\left|\mathcal{C}_{r}(n)\right|^{2}\right]+E\left[\left|\mathcal{C}_{j}(n)\right|^{2}\right]+E\left[|\mathcal{N}(n)|^{2}\right]}{\left(1-\frac{\pi \beta_{r} T_{s}}{3}\right)\left(1-\frac{\pi \beta_{j} T_{s}}{3}\right)\left[\left(\frac{E_{s}}{2 N_{0}}\right)\left(\left|H_{0,1}(n)\right|^{2}+\left|H_{1,0}(n)\right|^{2}\right)+1\right]}\right) .
\end{aligned}
$$

By increasing the transmitted power, the total composite noise increases due to the ICI at relay given by (15). In the expression for the ICI at destination given by (16), the first term is caused by a CPE term at relay while the second and the third terms are due to the ICI at relay and AWGN enhanced by energy of a PN term. In the high SNR region, the first and the second terms in the above expression drastically increase causing further performance degradation due to the PN and consequently, limits the system's performance. By analyzing (16) and (15), we observe that the ICI at destination includes the ICI at relay just enhanced by the energy of a PN term. Thus, the ICI at the relay has the highest impact on the total composite noise variance given by (17). The SINR degradation given by (20) directly depends on the composite noise variance. Due to that, among all terms in composite noise variance, the ICI at the destination has highest impact on the SINR degradation.

\subsection{BER}

In what follows, we evaluate the BER performance of ANC scheme in the presence of PN. We note here that the following analysis is valid only for data-modulation resulting with unit modulus data-symbols (i.e., $\left|d_{j}(n)\right|=1$ ). The conditional BER can be represented as [25]

$$
\begin{aligned}
p_{b}\left(\frac{E_{s}}{N_{0}},\left\{H_{m, j}(n)\right\}, \beta_{o}\right)= & \frac{1}{2} \operatorname{erfc} \\
& \times\left[\sqrt{\frac{1}{4} \gamma\left(\frac{E_{s}}{N_{0}},\left\{H_{m, j}(n)\right\}, \beta_{o}\right)}\right],
\end{aligned}
$$

where $\operatorname{erfc}[\cdot]$ denotes the complementary error function [25] and $m$ denotes the transmission phase (i.e., $m=$ $\{0,1\})$. The average BER is numerically evaluated by averaging (21) over all possible realization of $\left\{H_{m, j}(n) ; n=\right.$ $\left.0, \ldots, N_{c}-1\right\}$ as

$$
\begin{aligned}
P_{b}\left[\frac{E_{s}}{N_{0}}\right]= & \int \cdots \int P_{b}\left[\frac{E_{s}}{N_{0}},\left\{H_{m, j}(n)\right\}, \beta_{o}\right] p\left[\left\{H_{m, j}(n)\right\}\right] \\
& \prod_{n} d H_{m, j}(n) .
\end{aligned}
$$

Theoretical average BER is evaluated using Monte Carlo numerical computation method as follows. First, the 
channel impulse response of each link for both phases is generated to obtain the corresponding channel gains $\left\{H_{m, j}(n)\right\}$. The equalization weights $\left\{w_{j}(n)\right\}$ are computed, and the conditional SINR expression given by (18) is computed. Then, the average BER is computed for the given set of channel gains $\left\{H_{m, j}(n)\right\}$ and this procedure is repeated for large number of times.

\subsection{Ergodic channel capacity}

Next, we evaluate the ergodic channel capacity in the presence of PN for OFDM-based ANC scheme. Ergodic capacity represents an ensemble average of the information rate over the channel distribution, and it is important since it defines a transmit rate irrespective of the channel realization.

If the channel is not known at transmitter, for the given average $E_{s} / N_{0}$, the channel gains for both phases $\left\{H_{m, j}(n)\right\}$, the ergodic capacity $C\left(E_{s} / N_{0},\left\{H_{m, j}(n)\right\}, \beta_{o}\right)$ of the $j$ th user $U_{j}$ can be computed as [25]

$$
\begin{aligned}
C & =E\left[C\left(E_{s} / N_{0},\left\{H_{m, j}(n)\right\}, \beta_{o}\right)\right] \\
& =\int_{0}^{\infty} \cdots \int_{0}^{\infty} C\left(E_{s} / N_{0},\left\{H_{m, j}(n)\right\}, \beta_{o}\right) f_{h} \prod_{n} d H_{m, j}(n),
\end{aligned}
$$

where $C\left(E_{s} / N_{0},\left\{H_{m, j}(n)\right\}, \beta_{o}\right)$ is the conditional channel capacity and $f_{h}=f_{h}\left[H_{m, j}(n)\right]$ denotes the Rayleigh probability density function normalized such that $\sum_{l=0}^{L-1} E\left[\left|h_{l, m, j}\right|^{2}\right]=1$. To date, the solution to the integral in (23) has not been found in the closed-form, so we resort to the numerical computational methods.

The ergodic capacity can be rewritten as

$$
\begin{aligned}
C\left(\frac{E_{s}}{N_{0}}\right)= & \frac{1}{N_{c}} \int_{0}^{\infty} \cdots \int_{0}^{\infty} \sum_{n=0}^{N_{c}-1} \log _{2}\left[1+\gamma\left(\frac{E_{s}}{N_{0}},\left\{H_{m, j}(n)\right\}, \beta_{o}\right)\right] f_{h} \\
& \prod_{n} d H_{m, j}(n) .
\end{aligned}
$$

The evaluation of the ergodic capacity is done by using the Monte Carlo numerical computational method as follows. First, a set of path gains $\left\{h_{m, j} ; l=0, \ldots, L-1\right\}$ is generated to obtain the channel gains $\left\{H_{m, j}(n) ; n=\right.$ $\left.0, \ldots, N_{c}-1\right\}$. This is followed by the computation of the equalization weights $\left\{w_{j}(n) ; n=0, \ldots, N_{c}-1\right\}$, and afterwards the SINR expression given by (18) is computed. Finally, the Ergodic capacity is evaluated by averaging (24) a sufficient number of times for different realization of the channel.

\section{Simulation results and discussion}

\begin{tabular}{|c|c|c|}
\hline \multirow{3}{*}{ Transmitter } & Data modulation & QPSK \\
\hline & Block (IFFT) size & $N_{C}=256$ \\
\hline & Gl & $N_{g}=32$ \\
\hline \multirow[t]{3}{*}{ Channel } & \multicolumn{2}{|c|}{$L=16$-path frequency-selective block } \\
\hline & \multicolumn{2}{|c|}{ Rayleigh fading } \\
\hline & FDE & ZF \\
\hline Receiver & Channel estimation & Ideal \\
\hline
\end{tabular}

Simulation parameters are summarized in Table 1. We assume OFDM frame length of $N_{c}=256$, GI length
Table 1 Numerical parameters

of $N_{g}=32$ and QPSK data modulation/demodulation. As for the channel, we assume $L=16$-path block Rayleigh fading channel. The maximum delay difference is less than the GI length since $\left(L<N_{g}\right)$, and all paths in any link are independent with each other, while $\left\{h_{l, m, j} ; l=0, \ldots, L-1\right\}$ are zero-mean independent complex variables with variance $E\left[\left|h_{l, m, j}\right|^{2}\right]=1 / L$. We have chosen $f_{D} T_{s}=0.0001$ for the normalized Doppler frequency (where $1 / T_{s}=1 /\left[T_{c}\left(1+N_{g} / N_{c}\right)\right]$ ) which corresponds to a terminal speed of $40 \mathrm{~km} / \mathrm{h}$ for a $2-\mathrm{GHz}$ carrier frequency and a transmission data rate of $1 / T_{s}=$ $100 \mathrm{Msymbols} / \mathrm{s}$. We assume PN at user's and relay's local oscillator modeled as described in Section 2.1. Ideal channel estimation and no path loss and shadowing is assumed.

\subsection{SINR degradation}

The SINR degradation of ANC in the presence of PN is illustrated in Figure 2 as a function of PN linewidth at the relay and destination, and average signal energy per bit-to-AWGN power spectrum density ratio $E_{b} / N_{0}$ $\left(=0.5 \times\left(1+N_{g} / N_{c}\right) \times\left(E_{s} / N_{0}\right)\right)$ as a parameter. It can

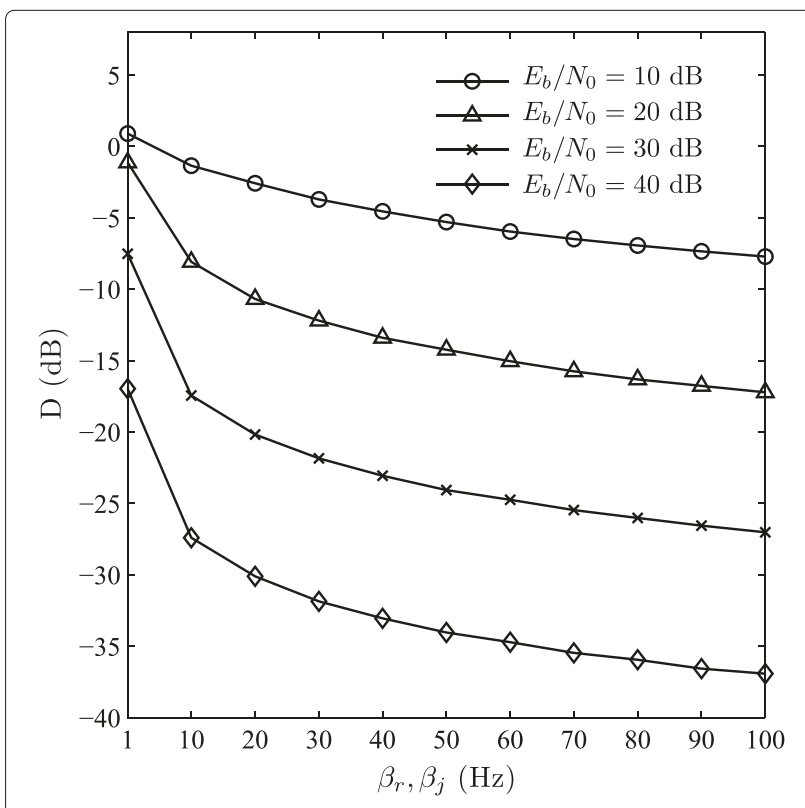

Figure 2 SINR degradation versus $\beta_{o}$. 
be seen from the figure that for $E_{b} / N_{0}=10 \mathrm{~dB}$, the SINR degradation is not evident since the SINR obtained in the presence of $\mathrm{PN}$ is just slightly lower in comparison to the SINR obtained in the case of absence of PN. However, as the power increases (i.e., $E_{b} / N_{0} \geq 20 \mathrm{~dB}$ ), the SINR degradation becomes more evident. This is due to the fact that increased emitted power cause higher contribution of the $\mathrm{CPE}$ and ICI terms in the variance of the composite noise. Our second observation is that for the PN linewidth values above $40 \mathrm{~Hz}$, SINR degradation remain within $5-\mathrm{dB}$ margin.

The SINR degradation as a function of $E_{b} / N_{0}$ and PN linewidth as a parameter is illustrated in Figure 3 . It is evident from the figure that the effect of PN becomes significant for the values of $E_{b} / N_{0}$ equals to $10 \mathrm{~dB}(20 \mathrm{~dB})$ corresponding to the PN linewidth of $1 \mathrm{~Hz}(10 \mathrm{~Hz})$. Interestingly, for $E_{b} / N_{0} \geq 20 \mathrm{~dB}$ we observe a considerably constant drift between the obtained SINR degradation curves.

\subsection{BER}

The average BER performance of ANC in the presence of $\mathrm{PN}$ as a function of average signal energy per bit-toAWGN power spectrum density ratio $E_{b} / N_{0}$ is illustrated in Figure 4. The average BER is obtained for the same PN linewidth at the relay and destination $\left(\beta_{r}=\beta_{j}=3 \mathrm{~dB}\right)$. We also plot the BER performance of ANC in the absence of $\mathrm{PN}$ for the comparison. It can be seen from the figure that the BER performance is more sensitive to $\mathrm{PN}$ at the destination, since the higher BER degradation is evident in comparison to the PN just at the relay. This is because

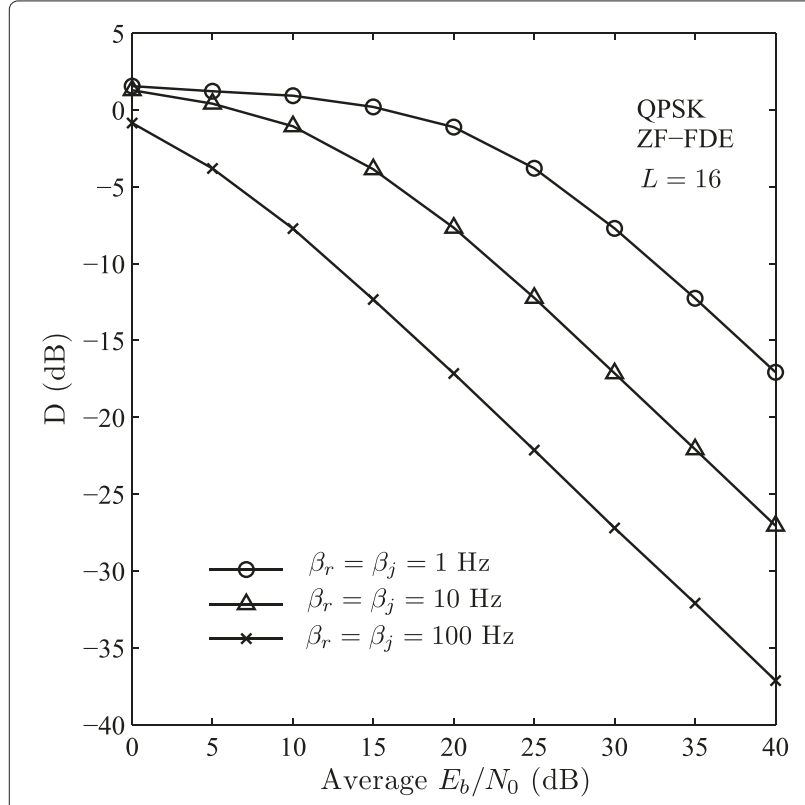

Figure 3 SINR degradation versus $E_{b} / N_{0}$.

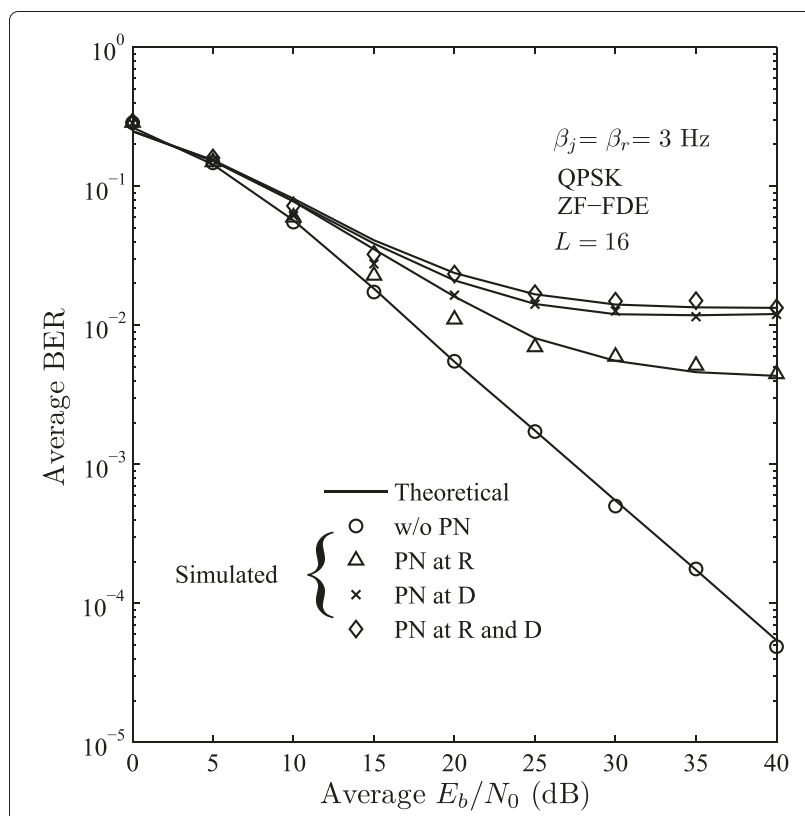

Figure 4 Average BER versus $E_{b} / N_{0}$.

the PN at the destination introduces higher ICI to the useful signal, and consequently the worse performance is obtained. The OFDM system becomes more sensitive on $\mathrm{PN}$ in the high SNR region, and this difference is more evident. A fairly good agreement between the theoretical and simulated results is achieved, which confirms the validity of the performance analysis presented in this article.

Next, the average BER performance as a function of PN linewidth for $E_{b} / N_{0}=30 \mathrm{~dB}$ is illustrated Figure 5. Once more we observe higher impact of $\mathrm{PN}$ at the destination in comparison to the PN at the relay. Moreover, the BER performance degradation is increasingly evident for the values bellow $20 \mathrm{~Hz}$. Beyond this PN linewidth value, the BER performance barely degrades since the contribution of corresponding introduced CPE and the ICI in the total composite variance significantly decreases.

\subsection{Ergodic capacity}

Here, we evaluate the impact of PN on ergodic capacity of the OFDM based ANC scheme. First, ergodic capacity as a function of the average signal energy per bit-to-AWGN power spectrum density ratio $E_{b} / N_{0}$, and PN linewidth as a parameter is plotted in Figure 6. We have plotted four different cases depending on which terminal the $\mathrm{PN}$ is introduced. It is evident from the figure that the ergodic capacity degradation due to the PN becomes significant for $E_{b} / N_{0} \geq 20 \mathrm{~dB}$. In the high SNR region, the first and the second terms in (16) (i.e., ICI at destination) corresponding to the CPE and ICI at relay enhanced by energy of a PN term drastically increase. Consequently, they cause further performance degradation and limit the 


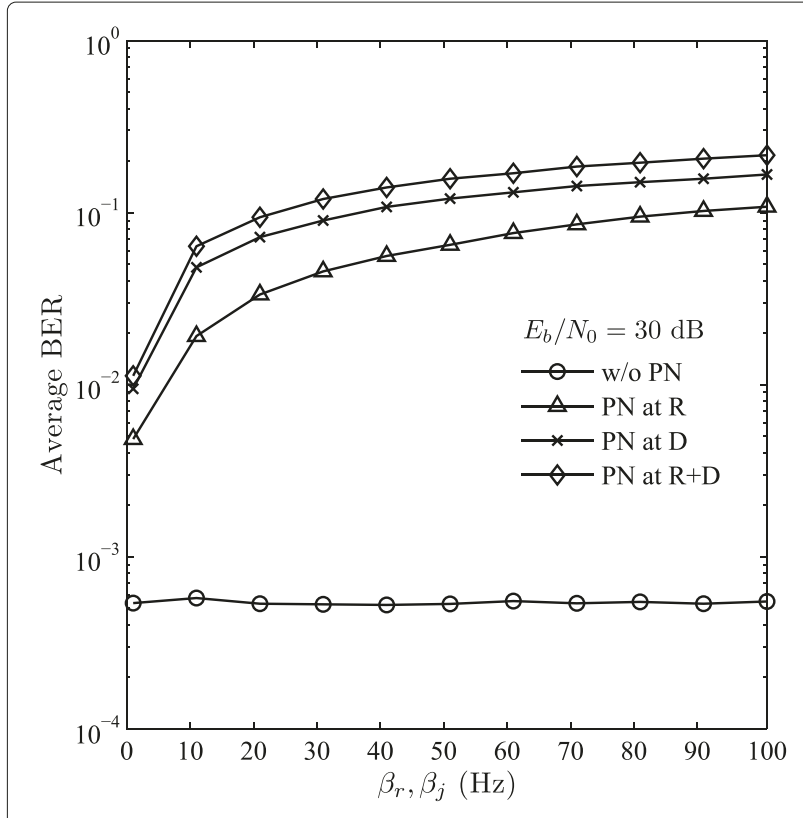

Figure 5 Average BER versus $\beta$.

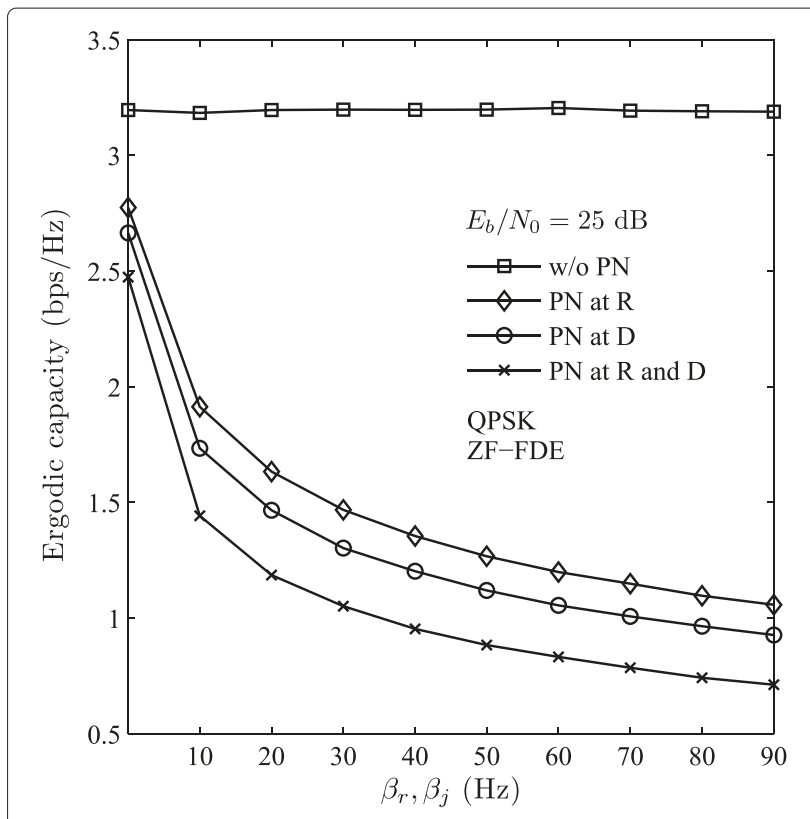

Figure 7 Ergodic capacity versus $\boldsymbol{\beta}_{\boldsymbol{o}}$. system's ergodic capacity. Moreover, the ergodic capacity is more sensitive to the $\mathrm{PN}$ encountered at the reception during the broadcast phase (i.e., at destination) in comparison to the reception during the multiple-access phase.

Then, we plot the ergodic capacity as a function of the PN linewidth in Figure 7. Clearly, the ergodic capacity decreases as the PN linewidth increases, and the

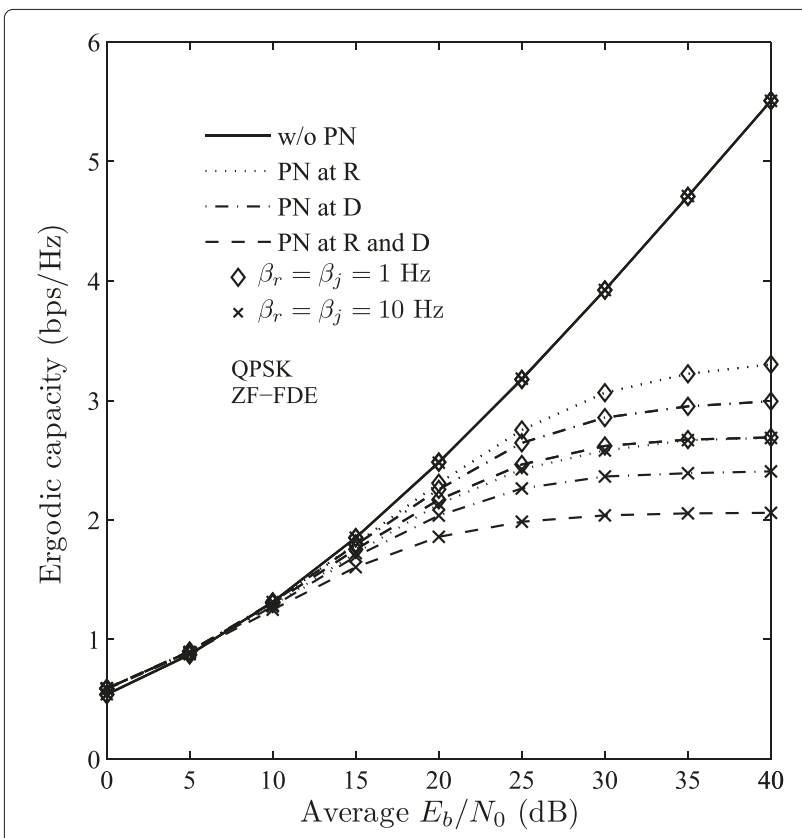

Figure 6 Ergodic capacity versus $E_{b} / N_{0}$. slope is particularly expressed for PN linewidth values in range (1-20) Hz. Furthermore, the difference between the obtained performance values is considerably constant $(0.15$ and $0.25 \mathrm{bps} / \mathrm{Hz})$ for $\mathrm{PN}$ linewidth values in range (25-90) Hz.

We underline that in this study, we have assumed perfect CSI. Imperfect CSI would further degrade the performance of ANC in the presence of PN, but it has been left out as interesting future study.

Based on the presented results, we conclude that implementation of the algorithms for mitigating the PN effects in ANC scheme should be done at the destination. Presented analysis should facilitate the design of such algorithms.

\section{Conclusion}

In this article, we theoretically analyze the impact of $\mathrm{PN}$ on the performance of ANC scheme using OFDM access in frequency-selective fading channel. Due to the property of ANC protocol, the PN is introduced during both, multiple-access phase (i.e., at relay) and broadcast phase (i.e., at destination). We derive the total composite noise variance of the ANC scheme in the presence of $\mathrm{PN}$ while approximating the PN as a zero-mean Wiener process to obtain the SINR expression. The average BER performance of ANC scheme is evaluated by the way of both, numerical and computer simulations. The performance is further evaluated through numerical simulation of the SINR degradation and ergodic channel capacity. Our results have shown that the performance of ANC scheme in terms of the average BER and ergodic capacity 
is more sensitive to the PN introduced during the broadcast phase, than during the multiple-access phase. This is because the PN at the destination introduces higher ICI to the useful signal and increases noise due to imperfect selfinformation removal leading to a inferior performance. Moreover, the SINR degradation increases as the transmitted power increases, which corresponds to the higher CPE and ICI contribution in the total composite noise. In addition, we observed rapid performance degradation of ANC scheme due to PN for the PN linewidth values up to $20 \mathrm{~Hz}$. Using analysis presented in this article, one may estimate the impact of phase noise during the first phase and accordingly design an iterative algorithm at the destination.

\section{Competing interests}

The authors declare that they have no competing interests.

\section{Acknowledgements}

This study was supported in part by the 2010 KDDI Foundation Research Grant Program.

\section{Author details \\ ${ }^{1}$ Communication Department, IPSA Institute, Sarajevo, Bosnia and Herzegovina. ${ }^{2}$ Motive Division, Alcatel-Lucent Bell, N.V., Antwerpen, Belgium. ${ }^{3}$ Department of Electrical and Communications Engineering, Tohoku University, Sendai, Japan.}

Received: 23 March 2012 Accepted: 27 November 2012

\section{Published: 2 January 2013}

\section{References}

1. S Katti, SS Gollakota, D Katabi, in ACM SIGCOM 2007. Embracing wireless interference: analog network coding, (Kyoto, Japan, August 2007), pp. 397-408

2. P Popovski, H Yomo, Wireless network coding by amplify-and-forward for bi-directional traffic flows, IEEE Commun. Lett. 11(1), 16-18 (2007)

3. S Zhang, S-C Liew, P Lam, in Proceedings of the ACM 12th MobiCom 2006. Hot topic: physical-layer network coding, (Los Angeles, USA, September 2006), pp. 358-365

4. H Gacanin, F Adachi, Broadband analog network coding, IEEE Trans. Wirel. Commun. 9(5), 1577-1583 (2010)

5. T Pollet, M Van Bladel, M Moeneclaey, BER sensitivity of OFDM systems to carrier frequency offset and Wiener phase noise, IEEE Trans. Commun. 43(234), 191-193 (1995)

6. L Tomba, On the effect of Wiener phase noise in OFDM systems, IEEE Trans. Commun. 46(5), 580-583 (1998)

7. SMallick, SP Majumder, in International Conference on Electrical and Computer Engineering 2008 (ICECE 2008). Performance analysis of an OFDM system in the presence of Carrier Frequency Offset, phase noise and timing jitter over rayleigh fading channels, (Dhaka, Bangladesh, December 2008), pp. 205-210

8. J Lee, HL Lou, D Toumpakaris, IEEE Global Telecommunications Conference 2005 (GLOBECOM '05), Analysis of phase noise effects on time-direction differential OFDM receivers. 5, 2674-2679 (December 2005)

9. SWu, Y Bar-Ness, OFDM systems in the presence of phase noise: consequences and solutions, IEEE Trans. Commun. 52(11), 1988-1996 (2004)

10. P Rabiei, W Namgoong, N Al-Dhahir, On the Performance of OFDM-based amplify-and-forward relay networks in the presence of phase noise, IEEE Trans. Commun. 59(5), 1458-1466 (2011)

11. MS El-Tanany, Y Wu, L Hazy, Analytical modeling and simulation of phase noise interference in OFDM-based digital television terrestrial broadcasting systems, IEEE Trans. Broadcast. 47, 20-31 (2001)
12. AG Armada, M Calvo, Phase noise and sub-carrier spacing effects on the performance of an OFDM communication system, IEEE Commun. Lett. 2, 11-13 (1998)

13. AG Armada, Understanding the effects of phase noise in orthogonal frequency division multiplexing (OFDM), IEEE Trans. Broadcast. 47, 153-159 (2001)

14. S Berger, A Wittneben, in IEEE Global Telecommunications Conference 2007 (GLOBECOM '07). Impact of noisy carrier phase synchronization on linear amplify-and-forward relaying, (Washington DC, USA, November 2007), pp. 795-800

15. L Lu, T Wang, SC Liew, S Zhang, in Proceedings of IEEE International Conference on Communications (ICC 2012). Implementation of physical-layer network coding, (Otawa, Canada, June 2012), pp. 4734-4740

16. D Sreedhar, A Chockalingam, in 2007 IEEE 18th International Symposium on Personal Indoor and Mobile Radio Communications (PIMRC). ICI-ISI mitigation in cooperative SFBC-OFDM with carrier frequency offset, (Athens, Greece, September 2007), pp. 1-5

17. $\mathrm{F} \mathrm{Ng}, \mathrm{X} \mathrm{Li}$, in Conference Record of the Thirty-Ninth Asilomar Conference on Signals, Systems and Computers, 2005. Cooperative STBC-OFDM transmissions with imperfect synchronization in time and frequency, (Pacific Groove, CA, USA, October 2005), pp. 524-528

18. H Gacanin, T Sjodin, F Adachi, On channel estimation for analog network coding in a frequency-selective fading channel, EURASIP J. Wirel. Commun. Netw. 2011 (2011)

19. G Wang, F Gao, X Zhang, C Tellambura, Superimposed training based joint CFO and channel estimation for CP-OFDM modulated two-way relay networks, EURASIP J. Wirel. Commun. Netw (SI on Physical Layer Network Coding for Wireless Cooperative Networks). 2010 (2010)

20. F Gao, R Zhang, Y-C Liang, Channel estimation for OFDM modulated two-way relay networks, IEEE Trans. Signal Process. 57(11), 4443-4455 (2009)

21. YG Li, GL Stuber, Orthogonal Frequency Division Multiplexing for Wireless Communications. (Springer, New York, 2006)

22. A Demir, A Mehrotra, J Roychowdhury, Phase noise in oscillators: a unifying theory and numerical methods for characterization, IEEE Trans. Circuits Syst. I. 47, 655-674 (2000)

23. E Meulen, Three-terminal communication channels, Adv. Appl. Probab. 3(1), 120-154 (1971)

24. A Sendonaris, E Erkip, B Aazhang, User cooperation diversity_-part I, II, IEEE Trans. Commun. 51(11), 1927-1948 (2003)

25. JG Proakis, Digital Communications, 3rd edn. (McGraw-Hill, New York, 1995)

doi:10.1186/1687-1499-2013-2

Cite this article as: Ligata et al.: On performance of analog network coding in the presence of phase noise. EURASIP Journal on Wireless Communications and Networking 2013 2013:2.

\section{Submit your manuscript to a SpringerOpen ${ }^{\circ}$ journal and benefit from:}

- Convenient online submission

- Rigorous peer review

- Immediate publication on acceptance

- Open access: articles freely available online

- High visibility within the field

- Retaining the copyright to your article

Submit your next manuscript at $>$ springeropen.com 